\title{
REAL TIME PROCESSING FOR EPIPOLAR RESAMPLING OF LINEAR PUSHBROOM IMAGERY BASED ON THE FAST ALGORITHM FOR BEST SCAN LINE SEARCHING
}

\author{
Xun Geng ${ }^{\mathrm{a}}$, Qing Xu ${ }^{\mathrm{a}}$, Shuai Xing ${ }^{\mathrm{a}, *}$, Chaozhen Lan $^{\mathrm{a}}$, Yifan Hou ${ }^{\mathrm{a}}$ \\ a Zhengzhou Institute of Surveying and Mapping, 450052 Zhengzhou, China - xing972403@163.com
}

WG II/2

KEY WORDS: Linear CCD, Differential rectification, Back projection of ground points, Best scan line searching, ADS40

\begin{abstract}
:
The epipolar resampling of linear pushbroom imagery is essential for automatic DEM extraction and feature collection. Due to the special imaging geometry of linear array scanners, the epipolar geometry of linear pushbroom imagery is very complex. In this paper, a fast algorithm for epipolar resampling of linear pushbroom imagery is proposed which can satisfy the real time processing demand of automatic DEM extraction and feature collection. The differential rectification of linear pushbroom imagery based on the rigorous geometric model is the basis for epipolar resampling. The back projection of ground points is essential for the differential rectification of linear pushbroom imagery and its main objective is searching the best scan line. The traditional best scan line searching methodology based on collinearity equation iterations needs a lot of calculation which has little practical use. According to the geometric constraints of the central perspective plane of scan line, the best scan line is determined efficiently within several iterations which just calculating the distance of ground point to the central perspective planar in object space. Based on our fast back projection algorithm, the epipolar resampling processing efficiency of linear pushbroom imagery can be improved greatly. The ADS40 airborne linear pushbroom imagery is tested and experiment results show that our algorithm has the merits of both high accuracy and efficiency which is suitable for real time processing of epipolar resampling.
\end{abstract}

\section{INTRODUCTION}

Due to the special imaging principle of linear CCD scanner, the epipolar geometry of linear pushbroom imagery is more complicated than that of frame image. Based on rigorous geometric model, Kim gives the epipolar line equation of linear imagery, but the equation is so complicated that it is very difficult to perform epipolar resampling according the epipolar line equation0. Morgan researched the epipolar resampling method of satellite linear imagery based on parallel projection0. Gong proposed a epipolar line image generation method for satellite image which is based on the RPC model0. In a word, many researhers has investigated this problem, however a general epipolar line model for linear pushbroom imagery is still under development. Based on former researchers' results, we know that (1)the epipolar line of linear pushbroom image is not a line but is a hyperbola line; (2)In local area, the epipolar line of linear pushbroom imagery can be seen as a line approximately; (3)In local area, epipolar line pairs exist which can make the image matching along the same row0.

In this paper, we proposed a new algorithm for epipolar resampling of linear pushbroom imagery in near real time based on a fast algorithm for best scan line searching. Experiment results demonstrate our method's feasibility and robustness.

\section{BASIC PRINCIPLE OF EPIPOLAR LINE FOR LINEAR PUSHBROOM IMAGERY}

For linear pushbroom imagery, we can't give the rigorous definition of epipolar line. The projection trajectory method is often used in the analysis of epipolar geometry of linear pushbroom imagery (See Figure. 1).

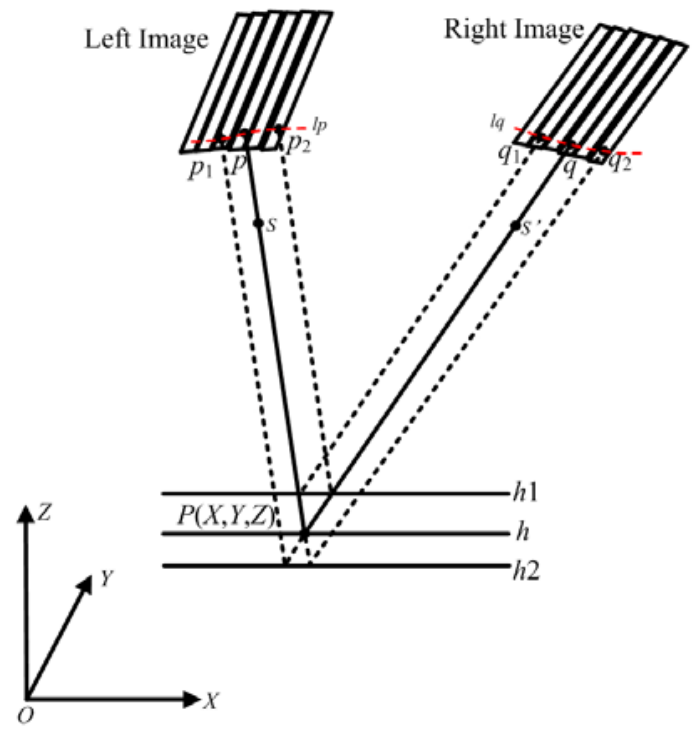

Figure 1. Epipolar geometry of linear pushbroom imagery based on projection trajectory

The basic principle of projection trajectory is as follows: the ground point is $P(X, Y, Z)$ and its two corresponding image points on left and right image are $p$ and $q$ respectively. Ground point $P$ and left image point $p$ can form a projection bundle line which through perspective center $S$. When the projection bundle line $p S P$ intersects with the horizontal plane in object space, a series of intersect points $P_{i}$ can be determined. Then $P_{i}$ is back projected onto right image and a series of image points $q_{i}$ can be determined which forms the epipolar curve lq. Obviously, $q$ which is the corresponding point of $p$ must lies in lq. In the

\footnotetext{
* Corresponding author.
} 
same way, the right image point $q$, perspective center $S^{\prime}$ and ground point $P$ forms the right bundle line and its projection trajectory on the left image can be determined.

The epipolar geometry equation of projection trajectory can be given as follows: a bundle line $q S$ can be determined through left image point $p\left(x_{l}, y_{l}\right)$ and perspective center $S\left(X_{\mathrm{si}}, Y_{\mathrm{si}}\right.$, $\left.Z_{\mathrm{si}}\right)$.

$$
\left[\begin{array}{l}
X \\
Y \\
Z
\end{array}\right]=\left[\begin{array}{l}
X_{s i} \\
Y_{s i} \\
Z_{s i}
\end{array}\right]+\lambda\left[\begin{array}{lll}
r_{11} & r_{12} & r_{13} \\
r_{21} & r_{22} & r_{23} \\
r_{31} & r_{32} & r_{33}
\end{array}\right]\left[\begin{array}{l}
x_{l} \\
y_{l} \\
-f
\end{array}\right]
$$

Where $r_{m n}(\mathrm{~m}, \mathrm{n}=1,2,3)$ is the rotation matrix coefficients, $\lambda$ is scale factor. Use the equation above, a series of ground point $P_{i}$ on $q S$ can be determind. Then $P_{i}$ is back projected onto the right image using the following equation:

$$
\begin{aligned}
& x_{r}=-f \frac{r_{11}^{\prime}\left(X-X_{s j}^{\prime}\right)+r_{21}^{\prime}\left(Y-Y_{s j}^{\prime}\right)+r_{31}^{\prime}\left(Z-Z_{s j}^{\prime}\right)}{r_{13}^{\prime}\left(X-X_{s j}^{\prime}\right)+r_{23}^{\prime}\left(Y-Y_{s j}^{\prime}\right)+r_{33}^{\prime}\left(Z-Z_{s j}^{\prime}\right)} \\
& y_{r}=-f \frac{r_{12}^{\prime}\left(X-X_{s j}^{\prime}\right)+r_{22}^{\prime}\left(Y-Y_{s j}^{\prime}\right)+r_{32}^{\prime}\left(Z-Z_{s j}^{\prime}\right)}{r_{13}^{\prime}\left(X-X_{s j}^{\prime}\right)+r_{23}^{\prime}\left(Y-Y_{s j}^{\prime}\right)+r_{33}^{\prime}\left(Z-Z_{s j}^{\prime}\right)}
\end{aligned}
$$

Where $r_{m n}^{\prime}(\mathrm{m}, \mathrm{n}=1,2,3)$ is rotation matrix coefficients of scan line $j$, $\left(X_{s j}^{\prime}, Y_{s j}^{\prime}, Z_{s j}^{\prime}\right)$ is the perspective center of scan line $j$. With equation (1) and (2), the following equation can be given

$$
\begin{aligned}
& x_{r}=-f \frac{r_{11}^{\prime} A+r_{21}^{\prime} B+r_{31}^{\prime} C}{r_{13}^{\prime} A+r_{23}^{\prime} B+r_{33}^{\prime} C} \\
& y_{r}=-f \frac{r_{12}^{\prime} A+r_{22}^{\prime} B+r_{32}^{\prime} C}{r_{13}^{\prime} A+r_{23}^{\prime} B+r_{33}^{\prime} C}
\end{aligned}
$$

where

$$
\begin{aligned}
& A=X_{s i}-X_{s j}^{\prime}+\lambda\left(r_{11} \cdot x_{l}+r_{12} \cdot y_{l}-r_{13} f\right) \\
& B=Y_{s i}-Y_{s j}^{\prime}+\lambda\left(r_{21} \cdot x_{l}+r_{22} \cdot y_{l}-r_{23} f\right) \\
& C=Z_{s i}-Z_{s j}^{\prime}+\lambda\left(r_{31} \cdot x_{l}+r_{32} \cdot y_{l}-r_{23} f\right)
\end{aligned}
$$

\section{REAL TIME PROCESSING FOR EPIPLOAR IMAGE}

For frame image the epipolar resampling can be performed by rectifying the original image onto the horizontal image. For the epipolar resampling of linear pushbroom imagery, we can use this concept too. On the other hand, after geometric rectified, the distortion of the linear pushbroom imagery is removed greatly which is useful for image matching. As shown in figure 2 , for the generation of epipolar image of linear pushbroom imagery, a virtual horizontal image is generated through geometric rectification. Then according to the analysis using projection trajection, the line direction of epipolar line can be determind and the approximate epipolar image can be acquired by resampling along the epipolar line.

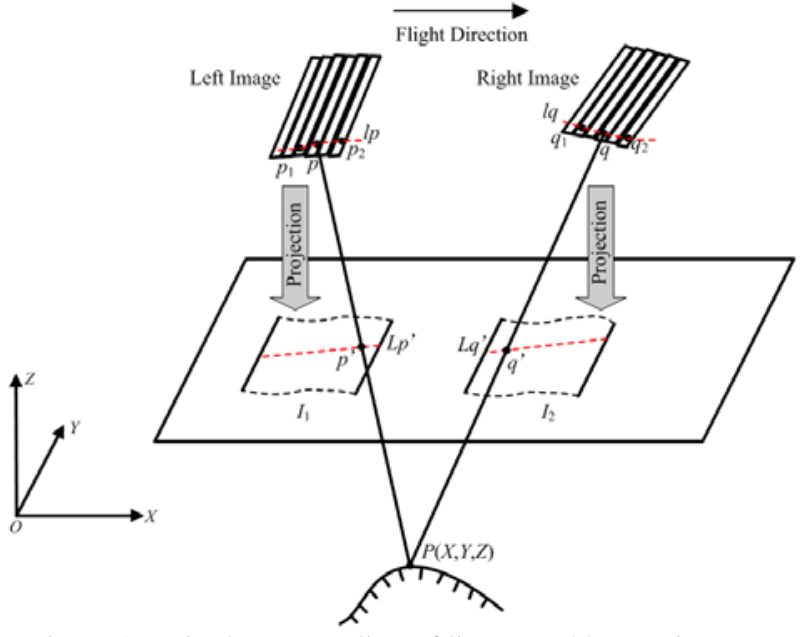

Figure 2. Epipolar resampling of linear pushbroom imagery based on virtual horizontal image

For airborne linear pushbroom image such as ADS40, the image size is very large and the generation of epipolar image may consume a lot of time. However, the epipolar image is just used for image matching or feature collection and it is not an image product. Therefore, we propose a method that generate epipolar image in memory in real time while there is no epipolar image stored in hard disk. The main computation for geometric rectification of linear pushbroom imagery focus on back projection and it is difficult to perform geometric rectification of linear pushbroom image in real time with traditional method. In this paper, we proposed a new back projection algorithm which can improve the efficiency of geometric rectification remarkably and consequently the epipolar resampling can be done in near real time. Here we use the geometric constraints of projection plane of each scan line in object space to improve the search speed of the best scan line. The computation is simple analytical calculation and the best scan line can be determined in several iterations. As shown in figure $3, S_{i}$ and $a b$ can construct a perspective projection plane. Given the average height, ground points $A$ and $B$ which correspondent to $a$ and $b$ respectively in image space can be calculated by collinearity equation. It is well known that a plane can be determined with three points.

$$
\left|\begin{array}{ccc}
x-x_{1} & y-y_{1} & z-z_{1} \\
x_{2}-x_{1} & y_{2}-y_{1} & z_{2}-z_{1} \\
x_{3}-x_{1} & y_{3}-y_{1} & z_{3}-z_{1}
\end{array}\right|=0
$$

Convert equation (6) to a general form

$$
A x+B y+C z+D=0
$$

The distance between a ground point $M\left(x_{0}, y_{0}, z_{0}\right)$ to the perspective projection plane can be calculated with following equation.

$$
d=\frac{\left|A x_{0}+B y_{0}+C z_{0}+D\right|}{\sqrt{A^{2}+B^{2}+C^{2}}}
$$

Through the geometric constraints of perspective projection plane of scan line, the perspective plane $i$ and $i+1$ which are nearest to the ground point $P$ can be found quickly. Consequently the best scan line can be determined accurately with following equation.

$$
L=i+\frac{D_{i}}{D_{i}+D_{i+1}}
$$




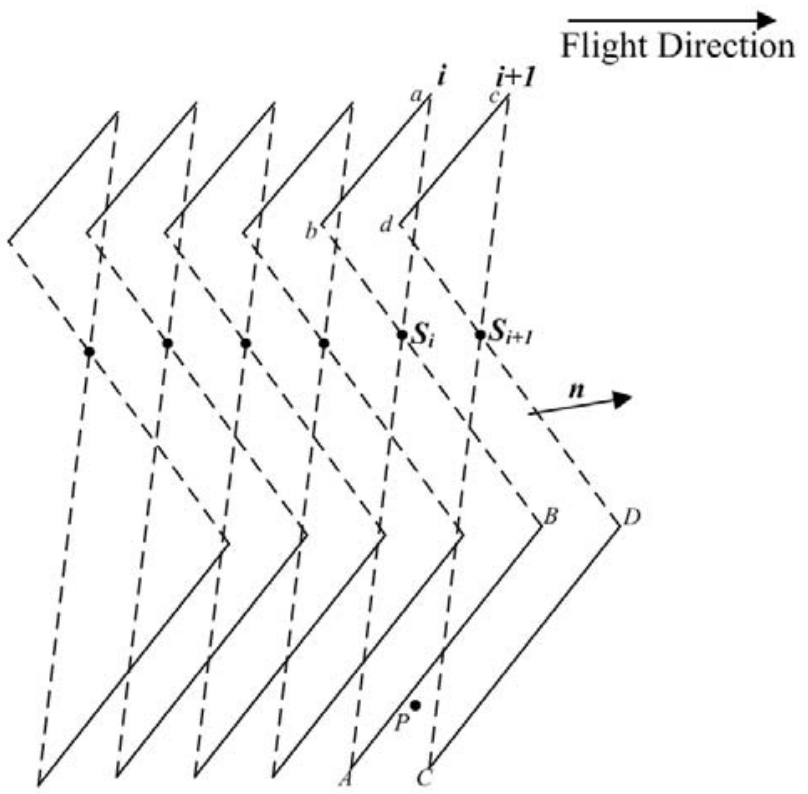

Figure 3. Fast algorithm for back projection

\section{EXPERIMENT RESULTS}

Experiments are tested on ADS40 airborne linear imagery. The GSD of raw ADS40 is about $35 \mathrm{~cm}$ and the flying height is about $4000 \mathrm{~m}$. Here we use two panchromatic channels of ADS40 that is PANF28 and PAN14 because they have a larger base to height ratio to form stereo image. After epipolar resampled and a red-green stereo image is generated as shown in figure 4 . The epipolar image size is about $1024 \times 768$ and the processing time is less than 0.5 second which can satisfy the real time processing demand. So the epipolar image can be generated in memory instead of in hard disk which can save both processing time and disk space. Ten corresponding points are measured on both PANF28 and PANB14 image to analyze the accuracy of epipolar image. As shown in table 1, the $y$ parallex is less than one pixel which demonstrates that our method's feasibility.

\section{CONCLUSIONS}

In this paper, a fast algorithm for epipolar resampling of linear pushbroom imagery is proposed which can satisfy the real time processing demand. Therefore, the epipolar resampling process can be saved and the epipolar image is generated in real time when automatic DEM extraction and feature collection is performed. Our method is based on the fast algorithm for best scan line searching which is the basis for data processing of linear pushbroom imagery. Experiment results demonstrate our method's feasibility.

\section{References}

Taejung Kim . A Study on the Epipolarity of Linear Pushbroom Image. Photogrammetry Engineer \& Remote Sensing, 2000, 66(8): 961-966.

Michel Morgan, Kyung-Ok Kim, Soo Jeong, and Ayman Habib. Epipolar Resampling of Space-borne Linear Array Scanner Scenes Using Parallel Projection. Photogrammetric Engineering \& Remote Sensing, 2006, 72(11): 1255-1263.
Gong Danchao, Tang Xiaotao, Zhang Li. The Transformation from Tilted Image to Horizontal Image of Line Camera Based on the Rational Function Model. Journal of Geomatics Science and Technology, 2012, 29(4): 240-243.

ZHANG Yongsheng, GONG Danchao, et al. The Application of High Resolution Satellite - the Imaging Model, Processing Algorithm and Application Technology. Beijing, Science Press, 2007, 16-23.

WANG Zhizhuo. Principles of Photogrammetry. Wuhan University Press, 2007:2-18.

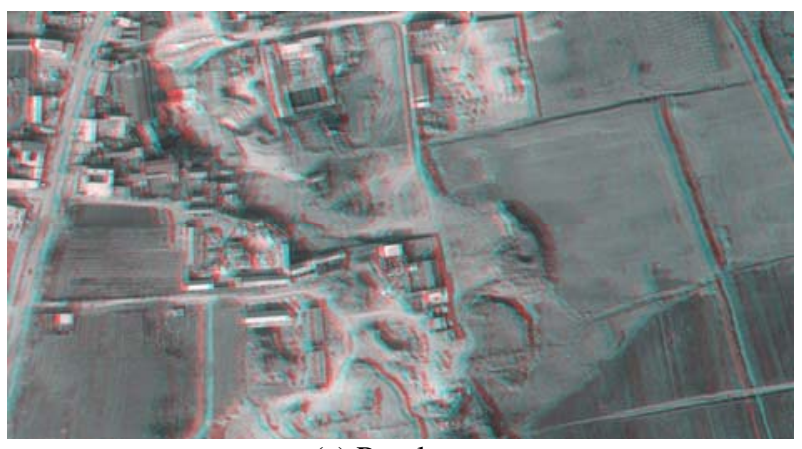

(a) Rural area

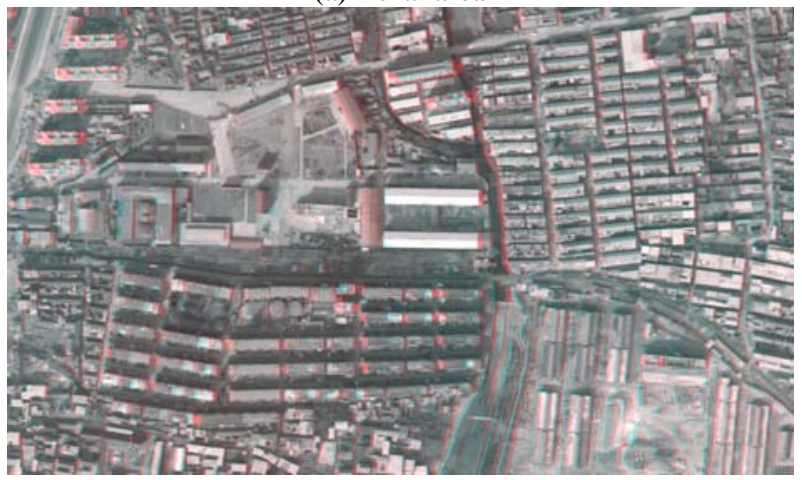

(b)City area

Figure 4. Experiment results

Table 1. Y papallex of corresponding points (pixel)

\begin{tabular}{|c|r|r|r|r|r|}
\hline Point ID & \multicolumn{1}{c|}{$x_{l}$} & \multicolumn{1}{c|}{$y_{l}$} & \multicolumn{1}{c|}{$x_{r}$} & \multicolumn{1}{c|}{$y_{r}$} & \multicolumn{1}{c|}{$d y$} \\
\hline 1 & 292.4 & 1107.1 & 302.4 & 1106.7 & -0.4 \\
\hline 2 & 310.8 & 1348.9 & 325.8 & 1348.6 & -0.3 \\
\hline 3 & 422.7 & 2089.3 & 435.7 & 2089.7 & 0.4 \\
\hline 4 & 810.8 & 1923.4 & 821.8 & 1922.8 & -0.6 \\
\hline 5 & 2108.2 & 4203.8 & 2127.2 & 4203.5 & -0.3 \\
\hline 6 & 3436.4 & 4510.9 & 3461.4 & 4511.3 & 0.4 \\
\hline 7 & 3890.4 & 5207.8 & 3922.4 & 5208.3 & 0.5 \\
\hline 8 & 5304.1 & 7123.3 & 5337.1 & 7122.5 & -0.8 \\
\hline 9 & 7192.6 & 8904.2 & 7213.6 & 8904.5 & 0.3 \\
\hline 10 & 8213.4 & 9018.4 & 8217.4 & 9019.1 & 0.7 \\
\hline
\end{tabular}

